PROCEEDINGS OF THE

AMERICAN MATHEMATICAL SOCIETY

Volume 136, Number 5, May 2008, Pages 1609-1617

S 0002-9939(08)08947-8

Article electronically published on January 8, 2008

\title{
SPECTRA OF OPERATORS WITH BISHOP'S PROPERTY $(\beta)$
}

\author{
M. DRISSI, M. EL HODAIBI, AND E. H. ZEROUALI
}

(Communicated by Joseph A. Ball)

\begin{abstract}
Let $X$ be a Banach space and let $\mathcal{A}(X)$ be the class that consists of all operators $T \in \mathcal{L}(X)$ such that for every $\lambda \in \mathbb{C}$, the range of $(T-\lambda I)$ has a finite-codimension when it is closed. For an integer $n \in \mathbb{N}$, we define the class $\mathcal{A}_{n}(X)$ as an extension of $\mathcal{A}(X)$. We then study spectral properties of such operators, and we extend some known results of multi-cyclic operators with $(\beta)$.
\end{abstract}

\section{INTRODUCTION}

The concept of quasisimilarity has been studied by many authors, and it is well-known that this concept generally does not conserve the spectral structure of an operator (see for example [1, 15]). Additional references including certain relationships between spectra of specific quasisimilar operators include [4, 6, 9] and 11. In this paper, for a Banach space $X$ and an integer $n \in \mathbb{N}$, we define classes $\mathcal{A}_{n}(X)$ and $\mathcal{N}_{n}(X)$ as an extension of the classes $\mathcal{A}(X)$ and $\mathcal{N}(X)$, respectively, introduced in [6]. Among other results we compare these classes, and we show that if $T \in \mathcal{A}_{n}(X)$ and $S \in \mathcal{A}_{m}(Y)$ are quasisimilar operators with Bishop's property $(\beta)$ on the Banach spaces $X$ and $Y$ for some tuple $(n, m) \in \mathbb{N}^{2}$, then $T$ and $S$ have the same approximate point spectrum, continuous spectrum and generalized spectrum and the same vein of other spectra.

\section{Preliminaries}

Throughout this paper, $X$ and $Y$ are Banach spaces and $\mathcal{L}(X, Y)$ denotes the space of all bounded linear operators from $X$ to $Y$. We set $\mathcal{L}(X):=\mathcal{L}(X, X)$ and for a bounded linear operator $A \in \mathcal{L}(X, Y)$, let $A^{*}, N(A)$, and $R(A)$ denote the adjoint operator, the null space, and the range of $A$. Also, we set $\alpha(A):=\operatorname{dim} N(A)$ and $\beta(A):=\operatorname{dim} N\left(A^{*}\right)$.

For an operator $T \in \mathcal{L}(X)$, we denote by $\operatorname{Lat}(T)$ the lattice of all closed $T$ invariant subspaces of $X$, and for $M \in \operatorname{Lat}(T)$, let $T \mid M \in \mathcal{L}(M)$ be the restriction of $T$ to $M$.

Received by the editors April 23, 2006 and, in revised form, September 18, 2006.

2000 Mathematics Subject Classification. Primary 47AXX, 47BXX.

Key words and phrases. Spectra, multi-cyclic operators, quasi-similar operators, Bishop's property $(\beta)$.

The research of the first and second authors was supported in part by a project of the Université Mohamed premier, Faculté des sciences, Oujda, Maroc.

(C)2008 American Mathematical Society 
For $X$ a Banach space, we define the class $\mathcal{A}(X)$ as the set of all operators $T \in \mathcal{L}(X)$ which satisfy the next relation

$$
\rho_{f}(T) \subseteq \rho_{\text {re }}(T),
$$

where $\rho_{f}(T)=\{\lambda \in \mathbb{C}: R(T-\lambda I)$ is closed $\}$ and $\rho_{\text {re }}(T)$ is the right essential spectrum. For $n \geq 1$ an integer, we denote by $\mathcal{A}_{n}(X)$ the set of all operators $T \in$ $\mathcal{L}(X)$ such that there exists a decreasing family of closed subsets $\left(X_{0}, \ldots, X_{n}\right) \in$ $\operatorname{Lat}(T)^{n+1}$ for which $X_{0}=X, T \mid X_{n} \in \mathcal{A}\left(X_{n}\right)$ and $T X_{i} \subseteq X_{i+1}$ for $i=0,1, \ldots, n-$ 1. Also, we set $\mathcal{A}_{0}(X):=\mathcal{A}(X)$. Clearly, the class $\mathcal{A}_{1}(X)$ contains all compact operators, but finite rank operators are not in $\mathcal{A}(X)$ for any infinite dimensional Banach space $X$. More examples are given in the following:

(1) If $X$ is finite dimensional, then every linear operator is in $\mathcal{A}(X)$.

(2) Every nilpotent operator $T$ of order $n$ is in $\mathcal{A}_{n}(X)$. Indeed, we can take $X_{i}=\operatorname{ker}\left(T^{n-i}\right)$. If $\operatorname{dim} X=+\infty$, then $T \notin \mathcal{A}(X)$.

(3) If $T$ is a normal operator such that every isolated point of $\sigma(T)$ is an eigenvalue of finite multiplicity, then $T$ is in $\mathcal{A}(X)$; see J.B.Conway [5], XI Propositions 4.5 and 4.6 .

We say that $T \in \mathcal{L}(X)$ has the single-valued extension property, abbreviated SVEP, if, for every open subset $U \subseteq \mathbb{C}$, the only analytic solution $f: U \longrightarrow X$ of the equation $(T-\lambda I) f(\lambda)=0$ for all $\lambda \in U$ is the zero function on $U$. Furthermore, we say that $T$ has Bishop's property $(\beta)$, if, for each open subset $U$ of $\mathbb{C}$ and every sequence of analytic functions $f_{n}: U \longrightarrow X$ for which $(T-\lambda I) f_{n}(\lambda) \rightarrow 0$ as $n \rightarrow \infty$, locally uniformly on $U$, it follows that $f_{n}(\lambda) \rightarrow 0$ as $n \rightarrow \infty$, again locally uniformly on $U$. For further details about these notions we refer to [2, [12] and [16].

Along this paper, we use $\sigma_{p}, \sigma_{a p}, \sigma_{e}$, and $\sigma_{S F}$ to denote the point, approximate point, Fredholm essential, and semi-Fredholm essential spectrum, respectively. We also denote by $\sigma_{l e}$ and $\sigma_{r e}$ the upper and lower semi-Fredholm essential spectra. Also, we use $\rho_{a p}, \rho_{e}, \rho_{S F}$, and $\rho_{l e}$ to denote the complement in $\mathbb{C}$ of $\sigma_{a p}, \sigma_{e}, \sigma_{S F}$ and $\sigma_{l e}$, respectively. Recall, when $T$ is a semi-Fredholm operator, the index of $T$ is defined as $\operatorname{ind}(T):=\alpha(T)-\beta(T)$.

Consider an arbitrary operator $T \in \mathcal{L}(X)$ and let $\sigma_{f}(T)$ consist of all $\lambda \in \mathbb{C}$ for which $(T-\lambda I)$ fails to have closed range, and let $\rho_{K}(T)$ consist of all $\lambda \in \mathbb{C}$ for which $R(T-\lambda I)$ is closed and $N(T-\lambda I) \subseteq R\left((T-\lambda I)^{n}\right)$ for all $n \in \mathbb{N}$. The Kato spectrum $\sigma_{K}(T):=\mathbb{C} \backslash \rho_{K}(T)$ is sometimes referred to as the semi-regular spectrum or the Apostol spectrum. Finally, we set $\rho_{f}(T):=\mathbb{C} \backslash \sigma_{f}(T)$.

Next, for $T \in \mathcal{A}_{n}(X)$, we set $T_{i}:=T \mid X_{i}$ for $i=1, \ldots, n$, and recall that, for $\sigma_{*} \in\left\{\sigma_{e}, \sigma_{r e}, \sigma_{l e}, \sigma_{S F}, \sigma_{a p}, \sigma_{f}\right\}$, from [3, Theorems 3, 5,6 and Example 11] we have $\sigma_{*}(T) \backslash\{0\}=\sigma_{*}\left(T_{1}\right) \backslash\{0\}$ and $\sigma_{*}\left(T_{i}\right) \backslash\{0\}=\sigma_{*}\left(T_{i+1}\right) \backslash\{0\}$ for $i=1, \ldots, n-1$. Consequently,

$$
\sigma_{*}(T) \backslash\{0\}=\sigma_{*}\left(T_{n}\right) \backslash\{0\} \quad \text { for } \quad \sigma_{*} \in\left\{\sigma_{e}, \sigma_{r e}, \sigma_{l e}, \sigma_{S F}, \sigma_{a p}, \sigma_{f}\right\} .
$$

Proposition 1.1 ([6, Theorem 4.1]). Let $T \in \mathcal{L}(X)$ and suppose that $T \in \mathcal{A}(X)$. Then the following statements hold:

(1) $\rho_{S F}(T)=\rho_{r e}(T)=\rho_{f}(T)$.

(2) $\rho_{e}(T)=\rho_{l e}(T)$.

(3) Moreover, if T has the SVEP, then

$$
\sigma_{e}(T)=\sigma_{S F}(T)=\sigma_{f}(T)=\sigma_{l e}(T)=\sigma_{r e}(T) .
$$


As an immediate consequence of Proposition 1.1 and (1.1) we get

Remark 1.1. For $T \in \mathcal{A}_{n}(X)$, we have

i) $\sigma_{r e}(T) \backslash\{0\}=\sigma_{f}(T) \backslash\{0\}$;

ii) $\sigma_{l e}(T) \backslash\{0\}=\sigma_{f}(T) \backslash\{0\}$ when $T$ has the SVEP.

Recall that a linear bounded operator with closed range is called normally solvable, and for an operator $T \in \mathcal{L}(X)$, the minimum modulus of $T$, written $\gamma(T)$, is defined by

$$
\gamma(T):=\inf \left\{\frac{\|T x\|}{\mathrm{d}(x, N(T))} \quad: \quad x \in X \backslash N(T)\right\}
$$

where $\mathrm{d}(x, N(T))$ denotes the distance from $x$ to $N(T)$ and with the convention that $\gamma(T)=\infty$ if $T=0$. Notice here that $T$ has closed range if and only if $\gamma(T)>0$. We refer to $[8]$ for further details and definitions.

Proposition 1.2. If $T \in \mathcal{A}_{n}(X)$, then the following assertions hold:

(i) If $R(T)$ is not closed, then $T \in \mathcal{A}(X)$.

(ii) If $T \in \mathcal{A}_{n}(X) \backslash \mathcal{A}(X)$, then $R(T)$ is closed and $\alpha(T)=\beta(T)=+\infty$.

Proof. (i) Since $T \in \mathcal{A}_{n}(X)$, it follows from (1.1) that

$$
\sigma_{r e}(T) \backslash\{0\}=\sigma_{f}(T) \backslash\{0\} .
$$

It is clear that, if $R(T)$ is not closed, then $\sigma_{r e}(T)=\sigma_{f}(T)$, which implies $T \in \mathcal{A}(X)$.

(ii) If $T \in \mathcal{A}_{n}(X) \backslash \mathcal{A}(X)$ using (i) $R(T)$ is closed. It is clear that $\beta(T)=+\infty$. Suppose that $\alpha(T)$ is finite; it will come that $T$ is normally solvable and has an index. Let $\lambda \in \mathbb{C}$ be given such that $0<|\lambda|<\gamma(T)$. From Theorem V.1.6 of [8], we get

$T-\lambda I$ is normally solvable $\quad ; \alpha(T-\lambda I) \leq \alpha(T) \quad$, and ind $(T-\lambda I)=$ ind $(T)$.

Hence $\lambda \in \rho_{f}(T)$ and (1.2) implies that $\lambda \in \rho_{\text {re }}(T)$. Consequently, $\alpha(T-\lambda I)$ and $\beta(T-\lambda I)$ are both finite. Finally it follows from the fact that $\alpha(T)$ is finite and ind $(T)=$ ind $(T-\lambda I)$ that $\beta(T)$ is finite. Contradiction, which completes the argument.

A straightforward application of Proposition 1.1 and Proposition 1.2, gives the following result

Theorem 1.2. Suppose that $T \in \mathcal{A}_{n}(X) \backslash \mathcal{A}(X)$. Then the following statements hold:

(1) $\sigma_{S F}(T)=\sigma_{r e}(T)=\sigma_{f}(T) \cup\{0\}$.

(2) $\sigma_{e}(T)=\sigma_{l e}(T)$.

(3) Moreover, if T has the SVEP, then

$$
\sigma_{e}(T)=\sigma_{S F}(T)=\sigma_{l e}(T)=\sigma_{r e}(T)=\sigma_{f}(T) \cup\{0\} .
$$

\section{The MAIN RESUlts}

In the following, for a subset $E$ of a Banach space, we set $\operatorname{span}\{E\}$ for the closure of the linear space generated by $E$ and $\bar{E}$ for the closure of $E$. We also denote $\mathbb{A}_{n}(X)$ for the set of all operators in $\mathcal{A}_{n}(X)$ such that $X_{i}:=\overline{R\left(T^{i}\right)}$ for $i=1, \ldots, n$. 
2.1. Quasisimilar operators in $\mathcal{A}_{n}(X)$. In this subsection, let $T \in \mathcal{L}(X)$ and $S \in \mathcal{L}(Y)$ be quasisimilar operators, equivalently, there exist $A \in \mathcal{L}(X, Y)$ and $B \in \mathcal{L}(Y, X)$ one to one and with dense ranges, such that $A T=S A$ and $T B=B S$. It is clear that, for each integer $n \geq 1, T^{n}$ and $S^{n}$ are quasisimilar operators. We set $\bar{T}_{n}:=T \mid \overline{R\left(T^{n}\right)}$ and $\bar{S}_{n}:=S \mid \overline{R\left(S^{n}\right)}$, and so, from Lemma 5.2 of [6], we obtain the following

Lemma 2.1. Let $T \in \mathcal{L}(X)$ and $S \in \mathcal{L}(Y)$ be quasisimilar operators; then $\bar{T}_{n}$ and $\bar{S}_{n}$ are quasisimilar operators.

Here we study the spectral picture of quasisimilar operators with property $(\beta)$.

Proposition 2.1. Suppose that $(T, S) \in \mathbb{A}_{n}(X) \times \mathbb{A}_{n}(Y)$ are quasisimilar operators with property $(\beta)$; then

$$
\sigma_{a p}(T)=\sigma_{a p}(S)
$$

Proof. Since $\bar{T}_{n}$ and $\bar{S}_{n}$ are quasisimilar operators by Lemma 2.1, and since $\bar{T}_{n}$ and $\bar{S}_{n}$ satisfy property $(\beta)$, it follows from Theorem 3.7 .15 of [12] together with (3) of Proposition 1.1, that

$$
\begin{gathered}
\sigma_{f}\left(\bar{T}_{n}\right)=\sigma_{f}\left(\bar{S}_{n}\right) \\
\sigma_{a p}\left(\bar{T}_{n}\right)=\sigma_{a p}\left(\bar{S}_{n}\right) .
\end{gathered}
$$

as $\sigma_{p}\left(\bar{T}_{n}\right)=\sigma_{p}\left(\bar{S}_{n}\right) ;$ thus

From this fact and (1.1), we deduce that

$$
\sigma_{a p}(T) \backslash\{0\}=\sigma_{a p}(S) \backslash\{0\} .
$$

Let us prove that $0 \in \sigma_{a p}(T)$ if and only if $0 \in \sigma_{a p}(S)$. Without loss of generality we can assume that $T$ and $S$ are not nilpotent operators (indeed, in this case, we have $\left.\sigma_{a p}(T)=\sigma_{a p}(S)=\{0\}\right)$.

Now, suppose that $0 \notin \sigma_{a p}(T)$, hence $0 \notin \sigma_{a p}\left(\bar{T}_{n}\right)$ and we get $0 \notin \sigma_{a p}\left(\bar{S}_{n}\right)$. It follows that $R\left(\bar{S}_{n}\right)$ is closed. Thus $\bar{S}_{n}$ is a Fredholm operator because $\bar{S}_{n}$ is injective and $\bar{S}_{n} \in \mathcal{A}\left(\overline{R\left(S^{n}\right)}\right)$, and so, $\bar{S}_{n}^{n}$ is Fredholm operator in $\overline{R\left(S^{n}\right)}$. Consequently, there exists a finite-dimensional subspace $F$ of $\overline{R\left(S^{n}\right)}$ such that

$$
\overline{R\left(S^{n}\right)}=R\left(\bar{S}_{n}^{n}\right) \oplus F .
$$

Since $R\left(\bar{S}_{n}^{n}\right) \subseteq R\left(S^{n}\right)$, it is easy to verify that

$$
R\left(S^{n}\right)=R\left(\bar{S}_{n}^{n}\right) \oplus\left(F \cap R\left(S^{n}\right)\right) .
$$

Therefore, $R\left(S^{n}\right)$ is closed, which implies that $S^{n}$ has a bounded inverse because $S^{n}$ is injective. Thus, $S$ also has a bounded inverse, and so $0 \notin \sigma_{a p}(S)$. Finally, the reverse implication is obtained by symmetry.

In what follows, let $T \in \mathcal{L}(X)$ and $S \in \mathcal{L}(Y)$ be quasisimilar operators, and let $A \in \mathcal{L}(X, Y)$ and $B \in \mathcal{L}(Y, X)$ be the quasi-affinities for which $A T=S A$ and $T B=B S$. In an easy way we refine Proposition 2.1 as follows

Theorem 2.1. Let $T$ and $S$ be quasisimilar operators with property $(\beta)$. Suppose that $T \in \mathcal{A}_{n}(X)$ and $S \in \mathcal{A}_{m}(Y)$ for some $(n, m) \in \mathbb{N}^{2}$. Then

(1) $\sigma_{*}(T)=\sigma_{*}(S) \quad$ for $\quad \sigma_{*} \in\left\{\sigma_{e}, \sigma_{r e}, \sigma_{l e}, \sigma_{S F}, \sigma_{a p}\right\}$.

(2) $\sigma_{f}(T) \cup\{0\}=\sigma_{f}(S) \cup\{0\}$.

Furthermore, if $\alpha(T)$ is finite, then $\sigma_{f}(T)=\sigma_{f}(S)$. 
Proof. Since $T$ and $S$ are with property $(\beta)$, Theorem 3.7 .15 of 12 together with the quasisimilarity of $T$ and $S$ imply that

$$
\sigma_{e}(T)=\sigma_{e}(S),
$$

and since Bishop's property implies the SVEP for an arbitrary bounded operator, Theorem 1.2 and Proposition 1.1 together with (1.2) implies that

$$
\sigma_{*}(T)=\sigma_{*}(S) \quad \text { for } \quad \sigma_{*} \in\left\{\sigma_{e}, \sigma_{r e}, \sigma_{l e}, \sigma_{S F}\right\}
$$

and

$$
\sigma_{f}(T) \cup\{0\}=\sigma_{f}(S) \cup\{0\} .
$$

If $\alpha(T)$ is finite, then $\alpha(S)$ is finite, and so, $(T, S) \in \mathcal{A}(X) \times \mathcal{A}(Y)$ by Proposition 1.2. Thus, $\sigma_{f}(T)=\sigma_{f}(S)$ by (2.1) and Proposition 1.1.

Now, let us prove $\sigma_{a p}(T)=\sigma_{a p}(S)$. It is known that

$$
\sigma_{a p}(T)=\sigma_{p}(T) \cup \sigma_{f}(T) \quad \text { and } \quad \sigma_{a p}(S)=\sigma_{p}(S) \cup \sigma_{f}(S),
$$

and it is clear that $\sigma_{p}(T)=\sigma_{p}(S)$. If $0 \in \sigma_{p}(T)$, then

$$
\begin{aligned}
\sigma_{a p}(T) & =\sigma_{p}(T) \cup \sigma_{f}(T) \cup\{0\} \\
& =\sigma_{p}(S) \cup \sigma_{f}(S) \cup\{0\} \\
& =\sigma_{a p}(S) .
\end{aligned}
$$

If, $0 \notin \sigma_{p}(T)$, then $\alpha(T)=0$ and so $\sigma_{f}(T)=\sigma_{f}(S)$. Hence by (2.2) we get $\sigma_{a p}(T)=\sigma_{a p}(S)$, which completes the proof.

Recall that, for $T \in \mathcal{L}(X)$, the continuous spectrum $\sigma_{c}(T)$ is defined to be the set of all $\lambda \in \mathbb{C}$ such that $(T-\lambda I)^{-1}$ exists but is not continuous and $R(T-\lambda I)$ is dense in $X$. Equivalently, $T$ is one to one and with nontrivial dense range (see for example [8]). It is then not hard to see that

$$
\sigma_{c}(T)=\sigma_{p}\left(T^{*}\right)^{c} \cap \sigma_{p}(T)^{c} \cap \sigma_{f}(T)
$$

where $K^{c}$ is the complement of the subset $K$ of $\mathbb{C}$. Also, it is known that $(T-\lambda I)$ is regular if $\lambda \in \rho_{K}(T)$ and there exist two closed subspaces $E_{\lambda}$ and $F_{\lambda}$ of $X$ such that

$$
X=N(T-\lambda I) \oplus E_{\lambda}=R(T-\lambda I) \oplus F_{\lambda} .
$$

Let, as usual, reg $(T)$ denote the regular set of $T$, and let $\sigma_{g}(T)$ denote to the generalized spectrum of $T$, which is the complement of reg $(T)$. We now extend the result of Theorem 2.1 to the continuous and the generalized spectrum.

Outlining the proof of [6, Lemma 5.2 ] we obtain the following result that will be needed in the next theorem.

Lemma 2.2. Let $T \in \mathcal{L}(X), S \in \mathcal{L}(Y)$ and $A \in \mathcal{L}(X, Y)$ be operators such that $A T=S A$ and $A$ has dense range. Then

$$
\overline{A \overline{R(T-\lambda I)}}=\overline{R(S-\lambda I)} \quad \text { for all } \quad \lambda \in \mathbb{C} .
$$

Theorem 2.2. Suppose that, $T \in \mathcal{A}_{n}(X)$ and $S \in \mathcal{A}_{m}(Y)$ for some $(n, m) \in \mathbb{N}^{2}$ are quasisimilar operators with property $(\beta)$. Then the following assertions hold:

(a) $\sigma_{c}(T)=\sigma_{c}(S)$,

(b) $\sigma_{g}(T)=\sigma_{g}(S)$. 
Proof. (a) First, let us prove that

$$
\sigma_{p}(T)^{c} \cap \sigma_{f}(T)=\sigma_{p}(S)^{c} \cap \sigma_{f}(S) .
$$

In fact, it is obvious that $\sigma_{p}(T)^{c}=\sigma_{p}(S)^{c}$. If $0 \notin \sigma_{p}(T)$, Theorem 2.1 implies $\sigma_{f}(T)=\sigma_{f}(S)$, and so, (2.4) holds. In the other case, when $0 \in \sigma_{p}(T)$, we have

$$
\begin{aligned}
\sigma_{p}(T)^{c} \cap \sigma_{f}(T) & =\sigma_{p}(T)^{c} \cap\left(\sigma_{f}(T) \cup\{0\}\right) \\
& =\sigma_{p}(S)^{c} \cap\left(\sigma_{f}(S) \cup\{0\}\right) \\
& =\sigma_{p}(S)^{c} \cap \sigma_{f}(S) .
\end{aligned}
$$

On the other hand, since $T^{*}$ and $S^{*}$ are quasisimilar operators, we get that $\sigma_{p}\left(T^{*}\right)^{c}$ $=\sigma_{p}\left(S^{*}\right)^{c}$. This, together with (2.3) and (2.4) entail the desired result.

(b) Since $T$ and $S$ have the SVEP, then from Corollary 3.1.7 of [12, we have $\rho_{K}(T)=\rho_{a p}(T)$ and $\rho_{K}(S)=\rho_{a p}(S)$, and from Theorem 2.1 we obtain

$$
\rho_{K}(T)=\rho_{K}(S) \text {. }
$$

Now, let $\lambda \in \operatorname{reg}(T)$ be given. Since $\lambda \in \rho_{K}(T)$ and from (2.5) we get $\lambda \in \rho_{K}(S)$; hence $R(S-\lambda I)$ is closed and $N(S-\lambda I)=\{0\}$. On the other hand, there exist two closed subspaces $E_{\lambda}=X$ and $F_{\lambda}$ of $X$ such that

$$
X=N(T-\lambda I) \oplus E_{\lambda}=R(T-\lambda I) \oplus F_{\lambda} .
$$

Suppose that $\lambda \neq 0$; Remark 1.1 i) together with $\lambda \in \rho_{f}(T)$ and $T \in \mathcal{A}_{n}(X)$ imply that $\lambda \in \rho_{r e}(T)$. Hence $F_{\lambda}$ is finite-dimensional, and thus $A F_{\lambda}$ is finite-dimensional. Hence,

$$
\begin{aligned}
Y=\overline{A X} & =\overline{A R(T-\lambda I) \oplus A F_{\lambda}} \quad(A \text { is injective }) \\
& =\overline{\overline{A R(T-\lambda I)}+A F_{\lambda}} \\
& =\overline{R(S-\lambda I)+A F_{\lambda}} \\
& =R(S-\lambda I)+A F_{\lambda},
\end{aligned}
$$

because $A F_{\lambda}$ is finite dimensional and $R(S-\lambda I)$ is closed; see [8]. Since $N(S-\lambda I)=$ $\{0\}$, then

$$
Y=N(S-\lambda I) \oplus Y
$$

Consequently, $\lambda \in \operatorname{reg}(S)$.

Now, suppose that $\lambda=0$, hence $0 \in \rho_{K}(T)$, and from $S V E P, \rho_{K}(T)=\rho_{a p}(T)$. It follows that $N(T)=\{0\}$, which implies $T \in \mathcal{A}(X)$ by Proposition 2.2. Thus $F_{0}$ is finite-dimensional, and therefore, for $\lambda \neq 0$, we have

$$
Y=R(S)+A F_{0}=N(S) \oplus Y .
$$

Consequently, $0 \in \operatorname{reg}(S)$. The reverse implication is obtained by symmetry.

We notice that this result is true for multi-cyclic operators and hence extends Theorem 5.6 of [11].

\section{ExAmples AND APPLiCATIONS}

Next, we shall apply the previous results to some classical classes of operators. 
3.1. Unilateral weighted shifts. Let $H$ be a Hilbert space, let $\left(e_{n}\right)_{n \in \mathbb{N}}$ be an orthonormal basis and let $\left(\omega_{n}\right)_{n \in \mathbb{N}}$ be a sequence of nonnegative numbers. The unilateral weighted shift $S_{\omega}$ associated with the sequence $\left(\omega_{n}\right)_{n \in \mathbb{N}}$ is the operator defined on the basis by $S_{\omega}\left(e_{n}\right)=\omega_{n} e_{n+1}$ for $n \geq 0$. It is well known that $S_{\omega}$ is bounded precisely when $w_{n}$ is a bounded sequence and that the spectrum is always a disc. Setting $r_{1}\left(S_{\omega}\right)=\lim _{n \rightarrow \infty} \inf _{k}\left(\omega_{n} \cdots \omega_{n+k}\right)^{1 / n}$, we have $\sigma_{f}\left(S_{\omega}\right)=$ $\left\{z \in \mathbb{C}\right.$, such that $\left.r_{1}\left(S_{\omega}\right) \leq|z| \leq r\left(S_{\omega}\right)\right\}$; here $r\left(S_{\omega}\right)$ stands for the usual spectral radius. For further information we refer to [14. Since from 14 all eigenvectors of $S_{\omega}^{*}$ are simple, it follows that $\sigma_{f}\left(S_{\omega}\right)=\sigma_{e}\left(S_{\omega}\right)=\sigma_{r e}\left(S_{\omega}\right)$ and hence that all shifts are in $\mathcal{A}$.

3.2. Rationally cyclic operators. Let $T$ be a bounded operator on an infinite dimensional Hilbert space $H$. T is said to be rationally cyclic if there exists $x \in H$ such that

$$
H=\{R(T) x: R \text { is a rational function with poles off } \sigma(T)\} .
$$

It is not difficult to see in this case that $\operatorname{dimker}(T-\lambda)^{*} \leq 1$ for any complex number $\lambda$; it follows that $T \in \mathcal{A}$. Now if we consider any power of $T$, it may fail to be rationally cyclic. However all results here apply to this setting.

3.3. Multi-cyclic operators. An operator $T \in \mathcal{L}(X)$ is called a multi-cyclic operator of order $n$ for some integer $n \geq 1$, abbreviated $n$-multi-cyclic, if there exist $n$ vectors $x_{1}, \ldots, x_{n} \in X$ such that $X=\operatorname{span}\left\{T^{k} x_{i} ; i=1, \ldots, n ; k \geq 0\right\}$ and if for every $n-1$ vector $z_{1}, z_{2}, \ldots, z_{n-1}$ in $X$, the subspace $\operatorname{span}\left\{T^{k} z_{i} ; i=1, \ldots, n-1 ; k \geq\right.$ $0\}$ is proper (see [10] and [7).

Now, set $\mathcal{N}_{0}(X)=\mathcal{M}(X)$ and for an integer $n \geq 1$, we define the class $\mathcal{N}_{n}(X)$ as the set of all operators $T \in \mathcal{L}(X)$ such that there exist an integer $m$ and $\left(x_{1}, x_{2}, \ldots, x_{m}\right) \in X^{m}$ for which

$$
R\left(T^{n}\right) \subseteq \operatorname{span}\left\{T^{n-1+k} x_{i} ; i=1,2, \ldots, m ; k \geq 0\right\} .
$$

It is not hard to see that for $T \in \mathcal{N}_{n}(X)$ there exist $\left(z_{1}, z_{2}, \ldots, z_{m}\right) \in X^{m}$ such that

$$
\overline{R\left(T^{n}\right)}=\operatorname{span}\left\{T^{k} z_{i} ; i=1,2, \ldots, m ; k \geq 0\right\} .
$$

So, the restriction of $T$ to $\overline{R\left(T^{n}\right)}$ is an $s$-multi-cyclic operator for some integer $s$, $1 \leq s \leq m$. The class $\mathcal{M}(X)$ consists of all operators $T \in \mathcal{L}(X)$ for which there is an integer $n$ such that $T$ is an $n$-multi-cyclic operator. Since $\mathcal{A}(X)$ contains $\mathcal{M}(X)$ (see [6]), it is clear that

$$
\mathcal{N}_{n}(X) \subseteq \mathbb{A}_{n}(X) \subseteq \mathcal{A}_{n}(X)
$$

We give some examples to show that the inclusions are strict for these classes of operators.

Let $X=H$ be a Hilbert space and $\left(e_{n}\right)_{n \geq 0}$ be an orthonormal basis of $H$. Let $\left(w_{n}\right)_{n \geq 0}$ be a bounded sequence of complex numbers. Recall that the unilateral weighted shift with weight $\left(w_{n}\right)_{n \geq 0}$ is given by

$$
\mathrm{T} e_{n}=w_{n} e_{n+1} \quad \text { for all } n \in \mathbb{N}
$$

and its adjoint operator is given by $\mathrm{T}^{*} e_{0}=0$ and $\mathrm{T}^{*} e_{n}=\bar{w}_{n-1} e_{n-1}$ for all $n \geq 1$. It is known that, if $\mathrm{T}$ is injective, we have that $R(\mathrm{~T})$ is closed if and only if the sequence $\left(\frac{1}{w_{n}}\right)_{n \geq 0}$ is bounded. For more details about weighted shifts see [14]. 
In the following examples, we consider $\mathcal{H}$ to be the Hilbert space given by $\mathcal{H}:=$ $H \oplus H$.

Example 1. Let $\mathrm{T}$ be the usual unilateral unweighted shift $\left(w_{n} \equiv 1\right)$ and $N$ be the unilateral weighted shift with $\left(w_{n}\right)_{n \geq 0}$ such that $w_{0}=1$ and $w_{n}=0$ for all $n \geq 1$. Set $L:=\mathrm{T} \oplus N$ as a bounded operator on $\mathcal{H}$, and so, $L^{*}=\mathrm{T}^{*} \oplus N^{*}$. Then, it is easy to verify that $L \in \mathcal{N}_{n}(\mathcal{H}) \backslash \mathcal{M}(\mathcal{H})$. On the other hand, $R(\mathrm{~T})$ is closed. Hence, $R(L)=R(\mathrm{~T}) \oplus \operatorname{span}\left\{e_{0}\right\}$ is closed. This together with the fact that $\alpha(L)$ and $\beta(L)$ are both infinite, implies that

$$
L \in \mathcal{N}_{n}(\mathcal{H}) \backslash \mathcal{A}(\mathcal{H}) \subseteq \mathcal{A}_{n}(\mathcal{H}) \backslash \mathcal{A}(\mathcal{H})
$$

Example 2. Consider $L$ defined as in Example 1 but $\mathrm{T}$ is the unilateral weighted shift with weight $\left(\frac{1}{n+1}\right)_{n \geq 0}$. Thus, $R(\mathrm{~T})$ is not closed and so $R(L)$ is not closed. This together with $L \in \mathcal{N}_{n}(\mathcal{H})$ and Remark $\left.2.1 \mathrm{i}\right)$, imply that

$$
L \in \mathcal{A}(\mathcal{H}) \backslash \mathcal{M}(\mathcal{H}) .
$$

Now, let $X$ be a Banach space, and with a similar proof of Proposition 5.1 of [6], we have the following proposition

Proposition 3.1. Let $T$ and $S$ be quasisimilar bounded operators; then $T \in \mathcal{N}_{n}(X)$ if and only if $S \in \mathcal{N}_{n}(Y)$.

As an immediate consequence of Proposition 1.2, Theorems 1.2, 2.1, 2.2, and $\mathcal{N}_{n}(X) \subseteq \mathcal{A}_{n}(X)$, we get the following corollaries.

Corollary 3.1. Let $T \in \mathcal{N}_{n}(X)$ with $\alpha(T)$ is finite; then $T \in \mathcal{A}(X)$. In particular, $T \in \mathcal{N}_{n}(X) \backslash \mathcal{A}(X)$ implies that $R(T)$ is closed, and $\alpha(T)$ and $\beta(T)$ are both infinite.

Corollary 3.2. Suppose that $T \in \mathcal{N}_{n}(X) \backslash \mathcal{A}(X)$. Then the following statements hold:

(1) $\sigma_{S F}(T)=\sigma_{r e}(T)=\sigma_{f}(T) \cup\{0\}$.

(2) $\sigma_{e}(T)=\sigma_{l e}(T)$.

(3) Moreover, if $T$ has the SVEP, then

$$
\sigma_{e}(T)=\sigma_{S F}(T)=\sigma_{l e}(T)=\sigma_{r e}(T)=\sigma_{f}(T) \cup\{0\} .
$$

Here $T \in \mathcal{N}_{n}(X)$ if and only if $S \in \mathcal{N}_{n}(Y)$ for all $n \in \mathbb{N}$. So, from the previous results we get

Corollary 3.3. Suppose that $T \in \mathcal{N}_{n}(X)$ and $S \in \mathcal{N}_{n}(Y)$ are quasisimilar operators. Then

$$
\sigma_{*}(T)=\sigma_{*}(S) \quad \text { for } \quad \sigma_{*} \in\left\{\sigma_{e}, \sigma_{r e}, \sigma_{l e}, \sigma_{S F}, \sigma_{a p}, \sigma_{c}, \sigma_{g}\right\}
$$

and $\sigma_{f}(T) \cup\{0\}=\sigma_{f}(S) \cup\{0\}$.

Furthermore, if $\alpha(T)$ is finite, then $\sigma_{f}(T)=\sigma_{f}(S)$.

\section{REFERENCES}

[1] J. Agler, E. Franks and D. A. Herrero, Spectral pictures of operators quasisimilar to the unilateral shift; J. Reine Angew. Math. 422 (1991) 1 - 20. MR.1133315 (92m:47007)

[2] E. Albrecht and J. Eschmeier, Analytic functional models and local spectral theory; Proc. London Math. Soc. 75 (1997) 323 - 348. MR 1455859 (98f:47043)

[3] B. A. Barnes, Common operator properties of the linear operators $R S$ and SR; Proc. Amer. Math. Soc. 126 (1998) 1055 - 1061. MR.1443814 (98f:47003) 
[4] L. Chen and Y. Zukin, Bishop's property $(\beta)$ and essential spectra of quasisimilar operators; Proc. Amer. Math. Soc. vol 128 (1999) $485-493$.

[5] J.B.Conway, A course in functional analysis; Graduate texts in Mathematics, 96, second edition, 1990. MR1070713 (91e:46001)

[6] M. Drissi and M. El Hodaibi, Spectra of quasisimilar operators; Submitted.

[7] M. EL Guendafi, M. Mbekhta and E. H. Zerouali, Bounded point evaluations for multicyclic operators, Banach Center Publ. 67 (2005) 199 - 217. MR2143926 (2006e:47012)

[8] S. Goldberg, Unbounded linear operators; Mc-Graw-Hill, New York, 1966 . MR0200692 $(34: 580)$

[9] D. A. Herrero, On the essential spectra of quasisimilar operators; Can. J. Math. 40 (1988)1436 - 1457. MR990108 (90b:47006)

[10] D. A. Herrero and L. Rodman, The multicyclic $n$-tuples of an n-multicyclic operator and analytic structures on its Spectrum; Indiana. Univ. Math. J. 34 (1985)619 - 629. MR794579 $(87 \mathrm{c}: 47025)$

[11] M. Mbekhta and E. H. Zerouali, Point d'évaluation pour les opérateurs cycliques ayant la propriété de Bishop $\beta$; Journal of Functional Anaysis 206 (2004)69 - 86. MR2024346 (2004i:47005)

[12] K. B. Laursen and M. M. Neumann, An introduction to Local spectral theory; London Mathematical Society Monograph, New series, Vol. 20, Clarendon Press, Oxford, 2000. MR 1747914 (2001k:47002)

[13] M.Schechter, On perturbations of essential spectra; J. London Math. Soc. 1, (1969)343 - 347. MR0248557(40:1809)

[14] A. L. Shields, Weighted shift operators and analytic function theory; Math. Surveys. Amer. Math. Soc. Providence 13(1974), 49 - 128. MR0361899 (50:14341)

[15] B. Sz-Nagy and C. Foias, Harmonic Analysis of Operators on Hilbert space, North HollandAmerican Elsevier, New York, 1970 (English edition). MR0275190 (43:947)

[16] F. H. Vasilescu, Analytic Functional Calculus and Spectral Decomposition, Editura Academiei, Bucharest, 1982. MR690957 (85b:47016)

Département de Mathématiques, Université Mohammed premier, Oujda, Maroc

E-mail address: m22drissi@yahoo.fr

Département de Mathématiques, Université Mohammed premier, Oujda, Maroc

E-mail address: hodaibi2001@yahoo.fr

Département de Mathématiques et Informatique, Université Mohammed V, BP 1014 RABat, Maroc

E-mail address: zerouali@fsr.ac.ma 